\title{
Regional Mechanical Thrombectomy Imaging Protocol in Patients Presenting with Acute Ischemic Stroke during the COVID-19 Pandemic
}

\author{
(D).S. Dhillon, (D) K. Pointon, (D) R. Lenthall, (D) S. Nair, (D) G. Subramanian, (D) N. McConachie, and (D). Izzath
} ow $=$

\begin{abstract}
BACKGROUND AND PURPOSE: Chest CT is a rapid, useful additional screening tool for coronavirus disease 2019 (COVID-19) in emergent procedures. We describe the feasibility and interim outcome of implementing a modified imaging algorithm for COVID19 risk stratification across a regional network of primary stroke centers in the work-up of acute ischemic stroke referrals for timecritical mechanical thrombectomy.
\end{abstract}

MATERIALS AND METHODS: We undertook a retrospective review of 49 patients referred to the regional neuroscience unit for consideration of mechanical thrombectomy between April 14, 2020, and May 21, 2020. During this time, all referring units followed a standard imaging protocol that included a chest CT in addition to a head CT and CT angiogram to identify Severe Acute Respiratory Syndrome coronavirus 2 (SARS-CoV-2) infective pulmonary changes.

RESULTS: Overall, 2 patients had typical COVID-19 radiologic features and tested positive, while 7 patients had indeterminate imaging findings and tested negative. The others had normal or atypical changes and were not diagnosed with or suspected of having COVID-19. There was an overall sensitivity of $100 \%$, specificity of $74.1 \%$, negative predictive value of $100 \%$, and positive predictive value of $22.2 \%$ when using chest CT to diagnose COVID-19 in comparison with the real-time reverse transcriptase-polymerase chain reaction test. The mean additional time and radiation dose incurred for the chest CT were $184 \pm 65.5$ seconds and $2.47 \pm 1.03$ mSv. Multiple cardiovascular and pulmonary incidental findings of clinical relevance were identified in our patient population.

CONCLUSIONS: Chest CT provides a pragmatic, rapid additional tool for COVID-19 risk stratification among patients referred for mechanical thrombectomy. Its inclusion in a standardized regional stroke imaging protocol has enabled efficient use of hospital resources with minimal compromise or delay to the overall patient treatment schedule.

ABBREVIATIONS: COVID-19 = coronavirus disease 2019; MT = mechanical thrombectomy; PPE = personal protective equipment; $\mathrm{PSC}=$ primary stroke centers; RT-PCR = real-time reverse transcriptase-polymerase chain reaction; SARS-CoV-2 = Severe Acute Respiratory Syndrome coronavirus 2

T he coronavirus disease 2019 (COVID-19), a manifestation of the Severe Acute Respiratory Syndrome coronavirus-2 (SARS-CoV-2), was declared a pandemic by the World Health Organization on March 11, 2020. ${ }^{1,2}$ At present, the COVID-19 incidence in the United Kingdom is one of highest in the world with 261,184 cases and 36,914

Received May 29, 2020; accepted after revision July 2.

From the Interventional Neuroradiology Department (P.S.D., R.L., S.N., N.M., W.I.), Queen's Medical Centre, Nottingham University Hospitals National Health Service Trust, Nottingham, UK; Cardiothoracic Radiology Department (K.P.) and Stroke Medicine Department (G.S.), Nottingham City Hospital, Nottingham University Hospitals National Health Service Trust, Nottingham, UK.

Please address correspondence to Permesh Dhillon, MD, Interventional NeuroRadiology Department, B Floor, Queens Medical Centre, Derby Road, Nottingham University Hospitals NHS Trust, Nottingham, NG7 2UH, United Kingdom; e-mail: permesh.dhillon@nhs.net

- Indicates open access to non-subscribers at www.ajnr.org

Indicates article with supplemental on-line appendix.

http://dx.doi.org/10.3174/ajnr.A6754 deaths, accurate as of May 25, 2020. ${ }^{3}$ Recent reports have described neurologic manifestations of COVID-19, including acute ischemic stroke. $^{4-6}$ Some of the proposed mechanisms underlying the increased prevalence of cardiovascular disease in COVID-19 include widespread systemic inflammatory and cytokine responses, diffuse intravascular coagulation, atherosclerotic plaque rupture, and hemodynamic alterations. $^{7-10}$

The latest publications by professional societies, including the Society of NeuroInterventional Surgery and the European Society of Minimally Invasive Neurological Therapy, stress the need for maintenance of services providing emergent mechanical thrombectomy (MT) in patients with cerebral large-vessel occlusion. ${ }^{11-13}$ The time-critical nature of MT precludes awaiting the results of any COVID-19 reverse transcriptase-polymerase chain reaction (RT-PCR) swab test, which, at present, can take up to 24 hours and has a false-negative rate, which currently mandates retesting to confirm negative status. 


\begin{tabular}{|c|c|c|}
\hline $\begin{array}{l}\text { COVID-19 Pneumonia } \\
\text { Imaging Classification }\end{array}$ & Rationale & CT Findings \\
\hline Typical appearance & $\begin{array}{l}\text { Commonly reported imaging } \\
\text { features of greater specificity } \\
\text { for COVID-19 pneumonia }\end{array}$ & $\begin{array}{l}\text { Peripheral, bilateral GGOs with or without consolidation or visible } \\
\text { intralobular lines (crazy-paving) } \\
\text { Multifocal GGOs of rounded morphology with or without consolidation } \\
\text { or visible intralobular lines (crazy-paving) } \\
\text { Reverse halo sign or other findings of organizing pneumonia (seen later in } \\
\text { the disease) }\end{array}$ \\
\hline Indeterminate & $\begin{array}{l}\text { Nonspecific imaging features of } \\
\text { COVID-19 pneumonia }\end{array}$ & $\begin{array}{l}\text { Absence of typical features AND } \\
\text { Presence of multifocal, diffuse, perihilar, or unilateral GGO with or } \\
\text { without consolidation lacking a specific distribution; they are } \\
\text { nonrounded or nonperipheral } \\
\text { Few very small GGOs with a nonrounded and nonperipheral distribution }\end{array}$ \\
\hline Negative & $\begin{array}{l}\text { Uncommonly or not reported } \\
\text { features of COVID-19 } \\
\text { pneumonia }\end{array}$ & $\begin{array}{l}\text { Absence of typical or indeterminate features AND } \\
\text { Presence of isolated lobar or segmental consolidation without GGO } \\
\text { Discrete small nodules (centrilobular, tree-in-bud sign) } \\
\text { Lung cavitation } \\
\text { Smooth interlobular septal thickening with pleural effusion } \\
\text { OR no abnormal findings }\end{array}$ \\
\hline
\end{tabular}

Note:-GGO indicates ground glass opacity.

${ }^{a}$ Adapted from the proposed Radiological Society of North America and British Society of Thoracic Imaging chest CT classification for reporting of COVID-19 pneumonia. 15,16

Patients with SARS-Cov-2 infection may be symptomatic or asymptomatic. Patients with acute ischemic stroke may not be able to provide a reliable clinical history or screening information due to underlying dysphasia or impaired consciousness. Additionally, family members may not be present to provide corroborating histories at the point of admission due to ongoing isolation and social distancing measures.

In combination, establishing COVID-related risks in this population is challenging. MT networks have to adopt a strategy for COVID-19 risk stratification to ensure the safety of healthcare professionals and other patients at the primary stroke centres (PSC), during inter-hospital ambulance transfer and during admission at the tertiary neuroscience unit.

A similar strategy is also required to select the intra-procedural anaesthetic technique, location of post-anaesthetic recovery and determine whether step-down care should be to a dedicated COVID-19 or non-COVID-19 ward. A pragmatic and rapid initial COVID-19 screening process within the PSC assists efficient use of personal protective equipment (PPE) and direction along the appropriate care pathway.

Herein, we describe our experience in a regional neuroscience center (Nottingham University Hospitals NHS Trust) that receives MT referrals from 6 PSCs, in implementing a coordinated regional approach to screening patients for both large-vessel occlusion and imaging evidence of COVID-19. We also undertook a retrospective review of the thoracic imaging to identify pulmonary and cardiovascular findings of clinical relevance.

\section{MATERIALS AND METHODS Study Population}

This study was registered with and approved by the audit committee of the hospital board, and individual patient consent was waived. We reviewed our prospectively collated data base of all hyperacute ischemic stroke referrals for consideration of MT between April 14, 2020 and May 21, 2020. Eligible patients were accepted for MT treatment based on local guidelines adapted from the National Institute of Clinical Excellence guidance. ${ }^{14}$

Following a regional protocol established before the pandemic, all hyperacute stroke referrals presenting with a suspected large-vessel occlusion within 24 hours of symptom onset routinely undergo an unenhanced CT of the head and dual-phase CT angiography from the aortic arch to the skull vertex. Following agreement with all referring PSCs, a chest CT was added to this protocol, to image the thorax. Referred patients being treated for COVID-19 during admission and those who had been tested within 48 hours before the referral did not warrant a chest CT.

Chest CT Parameters. Chest CT images were obtained on a Somatom Definition AS or AS Plus, 64- or 128-section multidetector CT system (Siemens) at our institution. The scan range was from the lung apices to the diaphragms immediately following acquisition of the dual-phase CT angiogram from the aortic arch to the skull vertex. We used the following reference acquisition parameters: tube voltage $=120 \mathrm{kV}$, tube current $=70 \mathrm{mAs}$, 0.5 -second helical rotation time, and 1.2 helical pitch. The reconstructed axial CT section thickness was $1 \mathrm{~mm}$, and further sections were reconstructed in the axial, coronal, and sagittal planes of $2-\mathrm{mm}$ section thickness at 2 -mm increments. The effective radiation dose calculated from the total dose-length product and the additional time incurred from completion of the dual-phase CT angiography to completion of the chest CT were obtained from the radiologic information system. There were variations of the scanner models and acquisition parameters across the regional hospitals.

\section{CT Reporting}

All images were reviewed by a neuroradiologist while considering the referral for MT. Chest CT findings were promptly reported by radiologists at the referring institutions to ensure optimization of detection and interpretation of findings, which may be subtle in early disease. The Radiological Society of North America and 


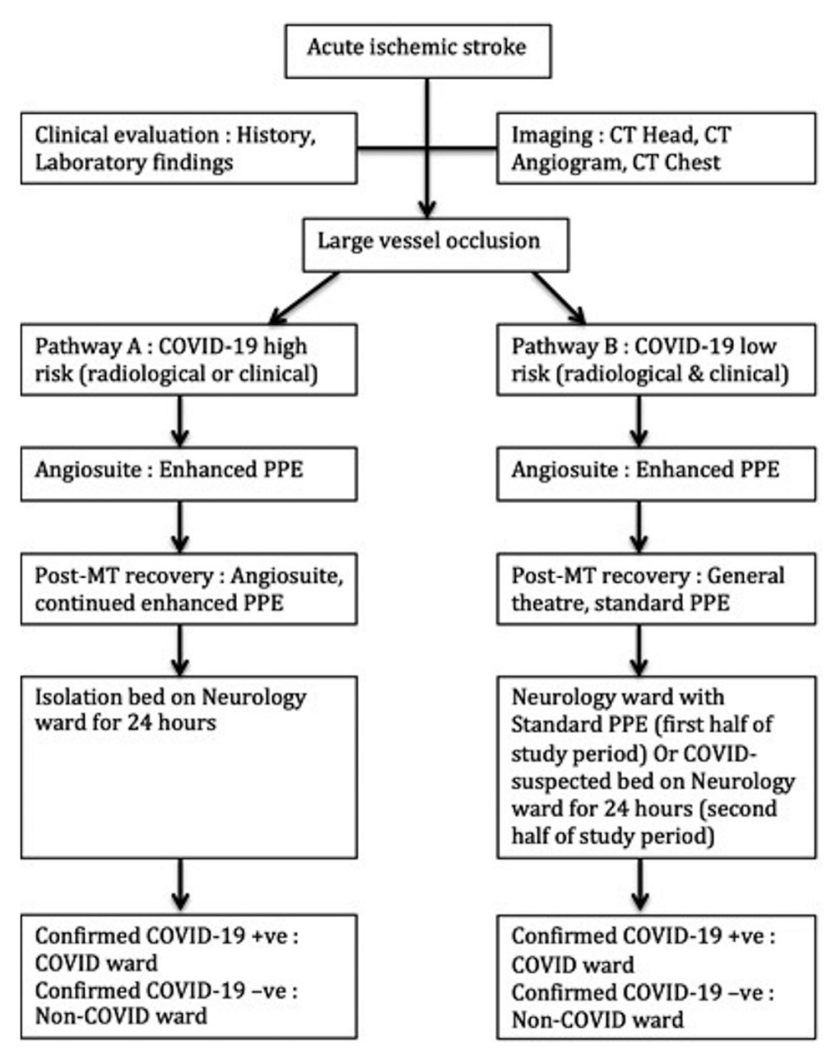

FIG 1. Comparison of the patient pathways according to the COVID19 risk. High risk for COVID-19 on admission included typical or indeterminate pulmonary findings on the chest CT and/or any clinical suspicion. Low risk for COVID-19 included atypical or normal findings on the chest CT and no clinical suspicion. Enhanced PPE indicates filtering facepiece class 3 masks, visors, and long-sleeved fluid-repellent overalls. Standard PPE indicates fluid-resistant surgical masks, gloves, and aprons; -ve, negative; +ve, positive.

the British Society of Thoracic Imaging publications of pulmonary findings in COVID-19 were used as a guide for the reviewing radiologist (Table 1). ${ }^{15,16}$ The thoracic imaging was then retrospectively reviewed by a cardiothoracic radiologist to audit thoracic CT findings using the British Society of Thoracic Imaging standardized evaluation to assess the presence and extent of COVID or other lung diseases. Rapid image transfer was enabled by the regional PACS network. All chest CT reports were reviewed on the local and regional PACS servers to identify the incidence of typical and indeterminate pulmonary features (deemed positive) or atypical and normal findings (deemed negative) for COVID-19. Discrepancies between the original thoracic CT reports and the retrospective review were communicated with the referring centers with further discussion planned at our local discrepancy meeting.

\section{Patient Pathway and Management}

Patients or their family members, if present and able, were asked a series of screening questions related to potential COVID-19 infection, and pertinent laboratory findings, including lymphopenia, were used while the patient was being worked-up for a potential MT (On-line Appendix). At the beginning of the study period, only patients who had any symptoms or pulmonary radiologic findings suspicious for COVID-19 underwent a nasopharyngeal or oral swab test. However, in the second half of the study period, all admitted patients underwent a swab test due to the gradually increasing availability of the test kits. The RT-PCR results were subsequently identified on the local institutions' clinical results reporting system, usually available within 24 hours. All patients accepted for MT were then transferred directly to our angiography suite for the procedure. Enhanced precautions were taken by all health care staff during the ambulance transfer and preparation of the angiography suite, peri- and intraprocedurally, particularly if any patient had suspected symptoms and/or had any typical or indeterminate radiologic findings of COVID-19 (Fig 1). Any suspicion of COVID-19 played a factor in the decision for procedures to be performed under conscious sedation or local or general anesthetic, made collaboratively by the interventional neuroradiologist and anesthetist. Intubation and extubation were performed within the negative pressure angiography suite. In view of the time pressures, the essential MT team members were locked in the angiography suite to avoid further delay associated with the mandated air exchange (between 10 and 30 minutes) after intubation. Appropriate deep cleaning and decontamination of the angiography suite were completed between cases. After MT, any patient with clinical and/or imaging features suspicious for COVID-19 was managed with additional precautions, with immediate post-general anesthetic recovery within the angiography suite as per institutional policy, which could take up to 2 hours. The patient was thereafter transferred to a dedicated COVID-19 bed according to our local protocol until he or she was fully worked-up clinically. In the latter half of the study period, separate COVID-19-proved beds and COVID19-suspected beds were established, and patients were transferred to the appropriate beds according to their known COVID-19 status at the time.

Standard data and statistical analysis was performed using GraphPad Prism (GraphPad Software).

\section{RESULTS}

Since the implementation of the revised imaging protocol during the study period, we received 49 MT referrals and performed 13 MTs. The mean age of all patients was $73.1 \pm 13.1$ years, with an age range of 52-102 years, with 25 men and 24 women. Among the patients undergoing MT, none had imaging features typical of COVID-19, while 3 patients had pulmonary changes indeterminate for COVID-19 but eventually tested negative. Two further patients had only clinical suspicion for COVID-19. All 5 patients were managed according to pathway A as described in Fig 1. The remaining 8 patients had no suspicious CT features and were not diagnosed with or suspected of having COVID-19 (managed according to pathway B). Different levels of PPE (enhanced PPE in high-risk patients and standard PPE in low-risk patients) were used during postanesthetic and ward care according to the COVID-19 risk.

Of the 36 patient referrals declined for MT, 2 patients had typical COVID-19 pulmonary features and tested positive, while 4 patients had indeterminate imaging findings and subsequently tested negative (Figs 2 and 3). The others had normal or atypical changes and were not diagnosed with or suspected of having 


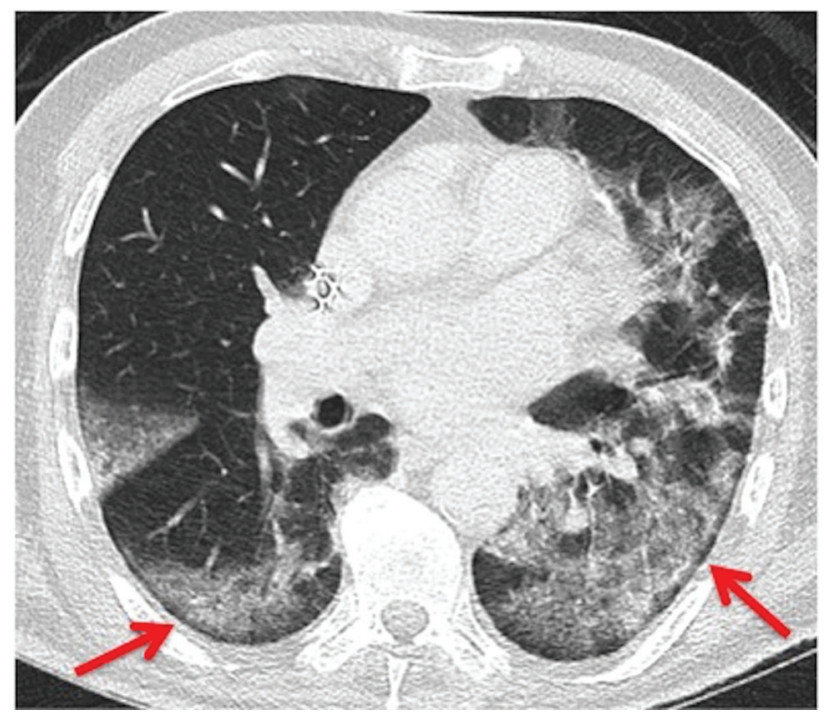

FIG 2. Typical features of COVID-19 pneumonia. Axial CT images at the level of the hilum show bilateral ground glass opacities (arrows) with areas of lobular sparing and sparing of the immediate subpleural area. This patient tested positive by RT-PCR analysis.

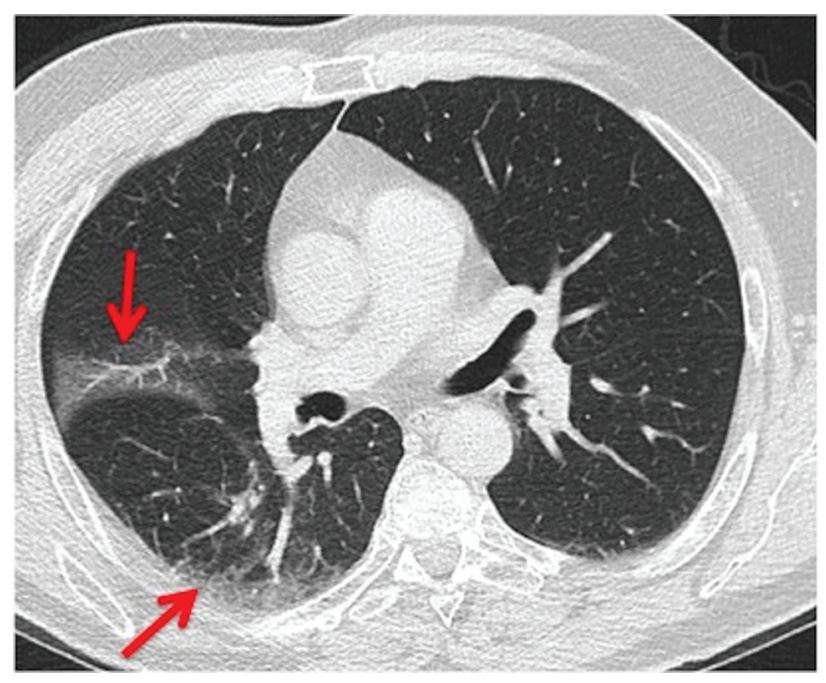

FIG 3. Indeterminate features of COVID-19 pneumonia. Axial CT images show unilateral, localized, peripheral ground glass opacities (arrows). This patient tested negative for COVID-19 by RT-PCR analysis.

COVID-19. Nine patients did not undergo a chest CT (tested/ diagnosed negative during or just before admission).

There was an overall sensitivity of $100 \%$, specificity of $74.1 \%$, negative predictive value of $100 \%$, and positive predictive value of 22.2\% when using chest CT to diagnose COVID-19 in comparison with the RT-PCR test (Table 2). The mean additional time incurred for the chest CT was $184 \pm 65.5$ seconds (range, 45-337 seconds). The average added effective radiation dose from the extension of the chest CT was $2.47 \pm 1.03 \mathrm{mSv}$.

There was good corroboration of the COVID-19 classification between general radiologists as primary reporters at the PSCs and the cardiothoracic radiologist. Only 1 case of indeterminate
Table 2: Correlation between chest CT features and RT-PCR swab results for the diagnosis of COVID-19 pneumonia ${ }^{a}$

\begin{tabular}{lll}
\hline & RT-PCR +ve & RT-PCR - ve \\
\hline Chest CT +ve & $2^{\mathrm{a}} / 0^{\mathrm{b}}$ & $6^{\mathrm{a}} / 1^{\mathrm{b}}$ \\
Chest CT -ve & $0^{\mathrm{a}} / 0^{\mathrm{b}}$ & $8^{\mathrm{a}} / 1^{\mathrm{b}}$ \\
\hline
\end{tabular}

Note:--ve indicates negative; +ve, positive.

${ }^{a}$ Clinical suspicion.

${ }^{\mathrm{b}}$ Asymptomatic for COVID-19 pneumonia.

Table 3: Comparison of classification of COVID-19 pulmonary findings between the primary reporter (general radiologist at primary stroke centers) and secondary reviewer (cardiothoracic radiologist) according to the adapted RSNA and BSTI guidance COVID-19

Pneumonia Imaging Primary Reporter Secondary Reviewer Classification $\quad(n=$ Patients $) \quad(n=$ Patients)

\begin{tabular}{lrr}
\hline Typical & 2 & 3 \\
Indeterminate & 7 & 7 \\
$\begin{array}{l}\text { Negative (atypical or } \\
\quad \text { normal) }\end{array}$ & 29 & 28 \\
\hline
\end{tabular}

Note:-BSTI indicates British Society of Thoracic Imaging; RSNA, Radiological Society of North America.

pulmonary features was more definitively labeled as typical, and 1 case of atypical findings was labeled as indeterminate by the cardiothoracic radiologist (Table 3). Multiple pulmonary and cardiovascular incidental findings, dichotomized according to clinical relevance, were identified following retrospective review of the thoracic imaging (Table 4).

\section{DISCUSSION}

This study describes the feasibility and outcome of implementing a modified imaging algorithm by the inclusion of a chest CT in the work-up of hyperacute stroke referrals for MT across a network of PSCs. Incidental thoracic findings of clinical relevance were also detected in this elderly cohort of patients with cardiovascular disease.

The recent publications from professional societies and review articles have suggested the possibility of inclusion of a complete chest CT in the scanning algorithm, but none have reported the value of it in a regional MT cohort. ${ }^{11-13,17,18}$ Kwee et $\mathrm{al}^{19}$ reported a series of incidental COVID-19 pulmonary findings in a small mixed cohort of patients presenting with acute ischemic stroke and head trauma. However, the incurred radiation dose and time of performing the additional chest CT could not be gleaned from their study.

Yang et $\mathrm{al}^{20}$ included chest CT in their local institution's COVID-19 screening and took enhanced precautions for COVID-19 MT patients, which resulted in overall delays of hospital arrival to puncture time in the MT cohort during the pandemic compared to a pre-pandemic period. In contrast to our study, we describe an attainable approach taken when faced with the additional challenge of implementing the revised triaging algorithm across all referring PSCs to avoid further delays on arrival at our neuroscience tertiary center.

The sensitivity, specificity, and negative predictive values reported are encouraging in our patient cohort and lend some support to findings from previous studies that have reported the 
Table 4: List of incidental findings identified on review of the thoracic imaging in this cohort, summarized according to their clinical relevance

Incidental Pulmonary/CVS Findings of Substantial

Clinical Relevance ( $\boldsymbol{n}=$ Patients)

Lung malignancy/suspicious nodule ${ }^{2}$

Pulmonary edema ${ }^{4}$

Left ventricular thrombus/aneurysm ${ }^{2}$

Left atrial dilation: anterior-posterior diameter $>45 \mathrm{~mm}^{16}$
Incidental Pulmonary/CVS Findings

of Indeterminate Clinical Relevance ( $n=$ Patients)

Emphysema $^{4}$

Lung fibrosis/pneumoconiosis ${ }^{3}$

Ascending aortic dilation ${ }^{2}$

Note:-CVS indicates cardiovascular.

potential benefit of chest CT in identifying features of COVID19 . $^{21-23}$ The poor positive predictive value of chest CT in our study is in line with previously reported studies. ${ }^{19,22}$ Chest CT has a high sensitivity for COVID-19 pneumonia, but its low-to-moderate specificity and positive predictive value preclude its use as a standalone screening tool for COVID-19 according to the recommendations by the Radiological Society of North America, the American College of Radiology, and the British Society of Thoracic Imaging. ${ }^{15,24}$ While the aim of this study was not to assess the use of chest CT as a stand-alone screening tool for COVID-19, chest CT may provide useful additional information in the context of time-critical interventions when the available clinical history may be limited and a COVID-19 lab test result is not available. Recent reports have described the need to ration PPE usage due to a global shortage in PPE supply. ${ }^{25}$ Furthermore, the lack of high sensitivity and specificity of the currently available tests, the extended turnaround time of obtaining these results, the lengthy postanesthesia recovery within the angiography suite, the decontamination procedure in patients with suspected COVID-19, and the shortage of critical care or isolation beds remain a challenge with a noticeable impact on health care and interventional services at our institution. Careful consideration of anesthetic choice should also be made due to the risks of airway intubation as an aerosol-generating procedure during general anesthesia, which is commonly used in MT cases. ${ }^{26}$ Hence, the addition of a screening chest CT during the work-up for emergent procedures, such as MT, may be of benefit in risk-stratifying this cohort of patients. ${ }^{27}$

The mean added time incurred with the inclusion of the chest CT was approximately 3 minutes in our study, which is in line with the proposed algorithm by Nguyen et al ${ }^{12}$ to consider chest CT if it did not incur more than a 5-minute delay to treatment. The mean radiation dose of $2.47 \mathrm{mSv}$ for the chest CT reported in our study falls below the average annual radiation dose per person in the UK of $2.7 \mathrm{mSv} /$ person/annum. ${ }^{28}$ The small addition of the radiation dose and scanning time incurred may be outweighed by the potential benefits of the outreaching effects on the patient management and safety of the involved health care staff.

In our study, no patient accepted for MT had typical radiologic findings or tested positive for COVID-19, while the minority of patients who had indeterminate radiologic findings or clinical suspicion for COVID-19 and those who did not have any suspicion for COVID-19, either symptomatically or radiologically, were managed according to our locally recommended PPE usage (detailed in the Fig 1 legend). Not all asymptomatic patients with normal imaging features were subsequently tested for COVID-19, particularly if they did not demonstrate an inflammatory response in their blood profile or have symptoms during admission, due to the limited availability of testing kits at the beginning of the study period. Hence, while unlikely, we cannot completely exclude the possibility that some of the aforementioned group of patients may be carriers of the SARS-CoV-2 virus.

The low incidence of patients with COVID-19 presenting with acute ischemic stroke symptoms during the study period could be due to the inclusion of asymptomatic patients, and the geographic variation in the rates of infection across our multiple localities compared with the more densely populated and highly endemic cities where increased infection rates have been reported. ${ }^{19,29}$ Nevertheless, the use of the suggested imaging algorithm is feasible and may be of further benefit in more endemic areas, where rapid COVID-19 testing kits are not available in the MT patient pathway. Kihira et $\mathrm{al}^{29}$ described incidental lung apical findings in patients with suspected COVID-19 during their routine imaging work-up using CT angiograms from the aortic arch for acute stroke presentations. However, as evidenced in previous studies, there is a possibility of missing a proportion of pulmonary findings that may only be prevalent in the mid to lower lung lobes, which are not covered in the routine imaging protocol. ${ }^{24,30}$

Following retrospective review of the thoracic imaging, multiple incidental findings were identified, which highlight the range of comorbidities present in a cohort of elderly patients with cardiovascular disease. In particular, we identified potential cardiac causes for underlying large-vessel occlusion, such as a left ventricular thrombus, as well as left atrial enlargement, known to be strongly associated with cardiac dysfunction and atrial fibrillation. ${ }^{31,32}$ Knowledge of these risk factors may be a factor that alters post-MT care or secondary prevention. Only half of these patients had known underlying cardiac disease such as cardiac failure, previous myocardial infarction, or atrial fibrillation. A proportion of findings also included pulmonary edema and cardiac failure, which may factor in decisions for the anesthetic choice in MT. Finally, a minority of patients had suspicious findings for underlying lung malignancy, which may have implications on their overall prognosis. There was minimal variation in the retrospective review of COVID-19 findings by the cardiothoracic radiologist and the primary reporters, which suggests satisfactory reporting of the COVID-19 classification with implications on the risk stratification across various PSCs.

Limitations of this study include its retrospective review and the inclusion of a relatively small cohort of patients. Additionally, this study only included patients referred with suspected largevessel occlusion for potential MT and does not encompass all acute suspected stroke referrals. RT-PCR testing of oro- or nasopharyngeal swabs was deemed the criterion standard for the diagnosis of COVID-19 in this study, despite known variations in its sensitivity. ${ }^{33}$ Also, there are limitations to the accuracy of chest CT in detecting COVID-19 in symptomatic and asymptomatic 
patients. Hence, correlation with the clinical findings, blood biomarkers, and RT-PCR results, whenever available, remains essential. Furthermore, there were variations in clinical practice during the study period due to changing local availability of and guidance on the PPE use, COVID-19 testing, and intensive care or ventilated beds. The COVID-19 status and the availability of ventilated beds also impacted the decision to accept MT referrals in elderly patients (older than 70 years of age) presenting with largevessel occlusion during the study.

\section{CONCLUSIONS}

For patients being referred for time-critical MT procedures in whom a reliable clinical history may not be available, chest CT provides a pragmatic, rapid additional tool that assists COVID-19 risk stratification, and its inclusion in a standardized stroke imaging protocol across our network of PSCs has enabled efficient use of hospital resources with minimal compromise or delay to the overall patient journey. The prevalence of incidental thoracic findings of clinical relevance in this patient cohort may be of benefit in the decision-making for future MT referrals and postprocedural care.

\section{ACKNOWLEDGMENTS}

We would like to thank the stroke, neurology, and radiology teams at the Nottingham University Hospitals National Health Service Trust, University Hospitals of Leicester National Health Service Trust, University Hospitals of Derby and Burton National Health Service Foundation Trust, Sherwood Forest Hospitals National Health Service Foundation Trust, and United Lincolnshire Hospitals National Health Service Trust.

Disclosures: Kate Pointon—UNRELATED: Employment: Nottingham University Hospital; Expert Testimony: legal reports; Royalties: chest x-ray educational book. Robert Lenthall—UNRELATED: Expert Testimony: Parliamentary Health Service Ombudsman, National Institute of Clinical Excellence, Comments: external advisor to the Parliamentary Health Service Ombudsman, expert advisor to National Institute of Clinical Excellence; Payment for Development of Educational Presentations paid to an individual: faculty member for The European Course in Minimally Invasive Neurological Therapy; Travel/Accommodations/Meeting Expenses Unrelated to Activities Listed: frequent recipient of expenses, Comments: personal expenses from many device manufacturers to support attendance at meetings during the past 2 decades, benefit from funding to professional interest groups to support such meetings; Other: past recipient of educational grants, Comments: In the past, Nottingham University Hospitals has received grants from MicroVention and Cordis Neurovascular to support an interventional neuroradiology fellowship training post, no current funding.* Norman McConachie—UNRELATED: Consultancy: MicroVention, Comments: for educational activity; Payment for Lectures Including Service on Speakers Bureaus: Stryker; Travel/Accommodations/Meeting Expenses Unrelated to Activities Listed: MicroVention. Wazim Izzath—UNRELATED: Payment for Lectures Including Service on Speakers Bureaus: Stryker, Comments: educational consultancy. *Money paid to the institution.

\section{REFERENCES}

1. Huang C, Wang Y, Li X, et al. Clinical features of patients infected with 2019 novel coronavirus in Wuhan, China. Lancet 2020;395:497506 CrossRef Medline

2. WHO Director-General's opening remarks at the media briefing on COVID-19 - 11 March 2020. https://www.who.int/emergencies/ diseases/novel-coronavirus-2019/interactive-timeline\#event-71. Accessed May 22, 2020
3. Coronavirus (COVID-19) in the UK. https://coronavirus.data.gov. $\mathrm{uk} /$ - category=regions\&map=rate. Accessed May 22, 2020

4. Li Y, Wang M, Zhou Y, et al. Acute cerebrovascular disease following COVID-19: a single center, retrospective, observational study. Stroke Vasc Neurol $2020 \mathrm{Jul}$ 2. [Epub ahead of Print] CrossRef Medline

5. Oxley TJ, Mocco J, Majidi S, et al. Large-vessel stroke as a presenting feature of Covid-19 in the young. N Engl J Med 2020;382:e60 CrossRef Medline

6. Goldberg MF, Goldberg MF, Cerejo R, et al. Cerebrovascular disease in COVID-19. AJNR Am J Neuroradiol 2020;41:1170-72 CrossRef Medline

7. Lillicrap D. Disseminated intravascular coagulation in patients with 2019-nCoV pneumonia. J Thromb Haemost 2020;18:786-87 CrossRef Medline

8. Klok FA, Kruip M, van der Meer NJM, et al. Incidence of thrombotic complications in critically ill ICU patients with COVID-19. Thromb Res 2020;191:145-47 CrossRef Medline

9. Smeeth L, Thomas SL, Hall AJ, et al. Risk of myocardial infarction and stroke after acute infection or vaccination. $N$ Engl $\mathrm{J} \mathrm{Med}$ 2004;351:2611-18 CrossRef Medline

10. Mehta P, McAuley DF, Brown M, et al; HLH Across Speciality Collaboration, UK. COVID-19: consider cytokine storm syndromes and immunosuppression. Lancet 2020;395:1033-34 CrossRef Medline

11. Fraser JF, Arthur AS, Chen M, et al. Society of NeuroInterventional Surgery recommendations for the care of emergent neurointerventional patients in the setting of Covid-19. J Neurointerv Surg 2020;12:539-41 CrossRef Medline

12. Nguyen TN, Abdalkader M, Jovin TG, et al. Mechanical thrombectomy in the era of the COVID-19 pandemic: emergency preparedness for neuroscience teams: a guidance statement from the Society of Vascular and Interventional Neurology. Stroke 2020; 51:1896-1901 CrossRef Medline

13. Aggour M, White P, Kulcsar Z, et al. European Society of Minimally Invasive Neurological Therapy (ESMINT) recommendations for optimal interventional neurovascular management in the COVID19 era. J Neurointerv Surg 2020;12:542-44 CrossRef Medline

14. Stroke and transient ischaemic attack in over 16s: diagnosis and initial management 2019. https://www.nice.org.uk/guidance/ng128. Accessed May 22, 2020

15. Simpson S, Kay FU, Abbara S, et al. Radiological Society of North America Expert Consensus Statement on Reporting Chest CT Findings Related to COVID-19: Endorsed by the Society of Thoracic Radiology, the American College of Radiology, and RSNA. J Thorac Imaging 2020;35:219-27 CrossRef Medline

16. BSTI CT reporting proforma: COVID-19 Version 2 13.04.2020. https://www.bsti.org.uk/media/resources/files/BSTI_COVID_CT_ Proforma_v2_13.04.2020.pdf. Accessed May 22, 2020

17. Leslie-Mazwi TM, Fargen KM, Levitt M, et al. Preserving access: a review of stroke thrombectomy during the COVID-19 pandemic. AJNR Am J Neuroradiol 2020;41:1136-41 CrossRef Medline

18. Smith MS, Bonomo J, Knight WA, et al. Endovascular therapy for patients with acute ischemic stroke during the COVID-19 pandemic: a proposed algorithm. Stroke 2020;51:1902-09 CrossRef Medline

19. Kwee RM, Krdzalic J, Fasen B, et al; COVID-19 CT Investigators South-East Netherlands (CISEN) Study Group. CT scanning in suspected stroke or head trauma: is it worth to go the extra mile and include the chest to screen for COVID-19 infection? AJNR Am J Neuroradiol 2020;41:1165-69 CrossRef Medline

20. Yang B, Wang T, Chen J, et al. Impact of the COVID-19 pandemic on the process and outcome of thrombectomy for acute ischemic stroke. J Neurointerv Surg 2020;12:664-68 CrossRef Medline

21. Dangis A, Gieraerts C, Bruecker YD, et al. Accuracy and reproducibility of low-dose submillisievert chest CT for the diagnosis of COVID19. Radiology: Cardiothoracic Imaging 2020;2:e200196 CrossRef

22. Ai T, Yang Z, Hou H, et al. Correlation of chest CT and RT-PCR testing in coronavirus disease 2019 (COVID-19) in China: a report of 1014 cases. Radiology 2020;296:E32-40 CrossRef Medline 
23. Fang Y, Zhang H, Xie J, et al. Sensitivity of chest CT for COVID-19: comparison to RT-PCR. Radiology 2020;296:E115-17 CrossRef Medline

24. Kim H, Hong H, Yoon SH. Diagnostic; performance of CT and reverse transcriptase-polymerase chain reaction for coronavirus disease 2019: a meta-analysis. Radiology 2020 Apr 17. [Epub ahead of Print] CrossRef Medline

25. Emanuel EJ, Persad G, Upshur R, et al. Fair allocation of scarce medical resources in the time of Covid-19. N Engl J Med 2020;382:2049-55 CrossRef Medline

26. Flexman AM, Abcejo AS, Avitsian R, et al. Neuroanesthesia practice during the COVID-19 pandemic: recommendations from Society for Neuroscience in Anesthesiology \& Critical Care (SNACC). J Neurosurg Anesthesiol 2020;32:202-09 CrossRef Medline

27. Qureshi AI, Abd-Allah F, Alsenani F, et al. Management of acute ischemic stroke in patients with COVID-19 infection: report of an international panel. Int J Stroke 2020;15:540-54 CrossRef Medline

28. Public Health England. Ionising Radiation Exposure of the UK Population: 2010 Review. https://www.phe-protectionservices.org. uk/cms/assets/gfx/content/resource_3595csc0e8517blf.pdf. Accessed May 22, 2020

29. Kihira S, Schefflein J, Chung M, et al. Incidental COVID-19 related lung apical findings on stroke CTA during the COVID-19 pandemic. J Neurointerv Surg 2020;12:669-72 CrossRef Medline

30. Wong HY, Lam HY, Fong AH, et al. Frequency and distribution of chest radiographic findings in COVID-19 positive patients. Radiology 2020;296:E72-78 CrossRef Medline

31. Stolzmann P, Scheffel H, Leschka S, et al. Reference values for quantitative left ventricular and left atrial measurements in cardiac computed tomography. Eur Radiology 2008;18:1625-34 CrossRef Medline

32. Takx RA, Vliegenthart R, Schoepf UJ, et al. Prognostic value of CT-derived left atrial and left ventricular measures in patients with acute chest pain. Eur J Radiol 2017;86:163-68 CrossRef Medline

33. Winichakoon $P$, Chaiwarith $R$, Liwsrisakun $C$, et al. Negative nasopharyngeal and oropharyngeal swabs do not rule out COVID-19. J Clin Microbiol 2020;58:e0297-20 CrossRef Medline 